\title{
Period Control of the Coupled Clock and Cell Cycle Systems
}

\author{
S. Almeida \\ Inria, iBV, Université Côte d'Azur \\ Sophia Antipolis, France \\ sofia.jf.almeida@gmail.com
}

\author{
M. Chaves* \\ Inria, Université Côte d'Azur, INRA, \\ CNRS, Sorbonne Université \\ Sophia Antipolis, France \\ madalena.chaves@inria.fr
}

\author{
F. Delaunay ${ }^{\dagger}$ \\ iBV, Université Côte d'Azur, CNRS, \\ INSERM \\ Sophia Antipolis, France \\ Franck.Delaunay@univ-cotedazur.fr
}

\begin{abstract}
The mammalian clock and cell cycle are two essential biological oscillators. In this work we investigate the coupling of these oscillators via non-linear dynamical modeling. We use previously developed reduced models of these systems and study a molecular interaction of MPF (mitosis promoting factor) repression by the CLOCK:BMAL1 protein complex, via induction of the repressor wee1. Furthermore, we propose an hypothesis whereby the clock responds to cell cycle Growth Factors (GFs) via a pathway involving the non-essential cell cycle complex cyclin D/cdk4 and study this interaction in the context of unidirectional clock $\rightarrow$ cell cycle coupling. We observe 1:1, 3:2, 4:3, 5:4 ratios of clock to cell cycle period and identify GF and the coupling strength $c_{b}$ as decisive control parameters for the system's state of synchronization. Synchronization ratios differing from 1:1, namely 3:2 and 5:4, have been observed in cells treated with the corticosteroid Dexamethasone (Dex). Here, we study Dex application and are able to reproduce the induction of ratios differing from 1:1. Finally, because slowing down the cell cycle is very relevant in the context of cancer therapies, we devise particular protocols of cell cycle period control with the use of clock inputs that are successful in substantially slowing down the cell cycle by the use of the system's synchronization dynamics, obtaining 2:3, 3:4, 4:5 ratios of clock to cell cycle period.
\end{abstract}

\section{CCS CONCEPTS}

- Applied computing $\rightarrow$ Biological networks; Systems biology; Bioinformatics;

\section{KEYWORDS}

Mammalian Cell Cycle, Circadian Cellular Clock, Coupled Oscillators, Period Control, Period-locking, Synchronization Ratios

\section{ACM Reference format:}

S. Almeida, M. Chaves, and F. Delaunay. 2019. Period Control of the Coupled Clock and Cell Cycle Systems. In Proceedings of The 10th International Conference on Computational Systems-Biology and Bioinformatics, Nice FRANCE, December 2019 (CSBio), 8 pages.

https://doi.org/10.1145/nnnnnnn.nnnnnnn

\footnotetext{
${ }^{*}$ http://www-sop.inria.fr/members/Madalena.Chaves/

${ }^{\dagger}$ http://ibv.unice.fr/research-team/delaunay/

Permission to make digital or hard copies of part or all of this work for personal or classroom use is granted without fee provided that copies are not made or distributed for profit or commercial advantage and that copies bear this notice and the full citation on the first page. Copyrights for third-party components of this work must be honored.

For all other uses, contact the owner/author(s)

CSBio, December 2019, Nice FRANCE

(C) 2019 Copyright held by the owner/author(s)

ACM ISBN 978-x-xxxx-xxxx-x/YY/MM...\$15.00

https://doi.org/10.1145/nnnnnnn.nnnnnnn
}

\section{INTRODUCTION}

The mammalian cell cycle and circadian clock are essential for cellular health and homeostasis. The cell cycle includes cellular growth and the division of a mother cell into two daughter cells (mitosis) and is a key mechanism of cellular renewal and tissue maintenance. The cellular circadian clock rhythmically controls patterns of gene activation and protein expression with a $24 \mathrm{~h}$ period, allowing the adaptation of cellular physiology to the daily environmental changes. The circadian clock impacts the majority of cellular genes and proteins, with genome-wide studies showing a large proportion of drug targets to exhibit circadian patterns of control [15]. As the mammalian clock and cell cycle often show synchronized rhythms in healthy cells they may be considered coupled oscillators.

Moreover, deregulation of any of these oscillators is linked to disease. A crucial example of unregulated cell cycle is that of certain cancers, where mutations induce accelerated and uncontrolled cellular division. In addition, deregulation of circadian rhythms is linked to inflammation and insulin resistance [12]. This type of deregulated systems presents evidence for the clock/cell cycle interconnection as, for instance, cancer is associated with circadian clock disturbances, inflammation and abnormal cellular metabolism [17]. Moreover, timed drug delivery, or chronotherapy, maximizes the desired drug effect while simultaneously minimizing undesired side-effects [15].

Firstly, observations of clock/cell cycle interconnection have revealed the entrainment of the cell cycle by the clock, for which some molecular mechanisms have been established. These are the induction of the MPF repressor wee 1 by the CLOCK:BMAL1 complex [13], the regulation of the cell cycle inhibitor p21 by the clock components REV-ERB- $\alpha / \beta$ and ROR- $\alpha / \gamma$ [9] and the clock-controlled repression of c-Myc [7], which is a promoter of cell cycle progression via cyclin $\mathrm{E}$ induction [16]. Examples of models describing an action of the clock on the cell cycle are Zámborszky et al., [22] and Gérard and Goldbeter, [8]. In this work we study clock $\rightarrow$ cell cycle coupling by modeling the CLOCK:BMAL1-mediated repression of MPF via wee1 induction.

More recently, however, evidence for a cell cycle action on the clock has been revealed. Notably, Feillet et al., [5] and Bieler et al., [4] have demonstrated in NIH3T3 mouse fibroblasts that the frequency of both clock and cell cycle increases for various values of $\%$ of FBS (Fetal Bovine Serum) in the medium. FBS contains Growth Factors (GFs) that are a mixture of nutrients and hormones known to accelerate the cell cycle. Observations reveal the clock to accelerate in the same manner as the cell cycle, so that the periods of both oscillators remain in a 1:1 ratio. Furthermore, the cell cycle 
mitotic event is verified to occur at a specific clock phase for all cells [5]. This is consistent with a model of oscillators that are phase-locked.

Phase locking (PL) is characterized by convergence of the combined phase of oscillation $\phi(t)=\left(\phi_{1}(t), \phi_{2}(t)\right)$ to a closed curve an attractor. Phase-locked oscillators are synchronized through the entire cycle - knowing the phase of one oscillator determines the phase of the other, in ideal noise-free systems. The observations of $1: 1$ phase-locking of clock and cell cycle $([4,5])$ have led to an hypothesis of a mechanism of cell cycle action on the clock, i.e. of the existence of bidirectional coupling between the oscillators. Studies of molecular interactions of cell cycle $\rightarrow$ clock coupling were made by Traynard et al., [18] and Almeida, [1]. By contrast, in this work we propose a different hypothesis: that of a clock that is responsive to Growth Factors (GFs). This is because unidirectional coupling can be sufficient for 1:1 phase-lock and observations show the increase of both clock and cell cycle frequencies with growth factor. As such, we derive a term for the GF $\rightarrow$ clock interaction, that is based on an established molecular pathway of cyclinD/CDK4 and PGC1- $\alpha$, [11].

Furthermore, Feillet et al., [5] observe that cells under the application of a pulse of Dexamethasone exhibit different synchronization ratios depending on the GF concentration. Dexamethasone (Dex) is a glucocorticoid agonist known to synchronize the clocks of cell populations. Synchronization ratios of clock to cell cycle periods in Dex-treated cells were determined to be approximately 5:4 for $10 \%$ FBS and 3:2 for 20\% FBS. Additionally, for 20\% FBS cells segregate in two groups with the second one maintaining 1:1 synchronization. In the same experiment a trimodal peak occurs for the distribution of mitosis with circadian clock phase [5], an observation that has also been made by Nagoshi et al., [14] under a similar protocol. Almeida, [1] have reproduced such synchronization ratios in both unidirectional cell cycle $\rightarrow$ clock and bidirectional coupling, under variation of different control parameters and when reproducing the Dex-treatment protocol in silico.

In this work we investigate the dynamics of mammalian clock and cell cycle coupling, using reduced dynamical models of both systems. The cell cycle model was developed and analyzed by Almeida et al., [3] and in its most reduced form consists of a two variable system forming a main negative regulatory loop between the essential cell cycle component MPF (mitosis promoting factor), which is the cyclin $\mathrm{B} / \mathrm{CDK} 1$ complex necessary and sufficient to carry out the mitotic process, and its repressor the APC:cdc20 complex. Furthermore, this model includes terms describing the action of CDC25 phosphatase, an MPF activator, and the WEE1 kinase, an MPF repressor. These terms are simplified as control loops of MPF on itself, thus including the regulation MPF exerts in regulating CDC25 and WEE1. This model yields relaxation oscillations and shows regions of bistability of active MPF with the amount of cell cycle GF [3]. The circadian clock model was developed by Almeida et al., [2] and is based on the major transcription translation feedback loop (TTFL) of the mammalian clock, where the protein complex CLOCK:BMAL1 promotes the PER and CRY proteins that form the PER:CRY complex, a repressor of CLOCK:BMAL1. This model is centered on the regulation that occurs via three types of DNA response elements within gene promoters: E-boxes, R-boxes and Dboxes, and includes the effect of activators and repressors on these boxes. It exhibits antiphasic oscillations between CLOCK:BMAL1 and PER:CRY, a characteristic of the mammalian circadian clock, and it has been used to study the interconnection between clock and metabolism [2]. In its reduced form, the clock model consists of 4 variables: BMAL1, REV, DBP and PER:CRY and has already been used by Almeida, [1] to study cell cycle $\rightarrow$ clock coupling as well as bidirectional coupling.

Our work aims at exploring strategies for cell cycle period control in the context of unidirectional clock $\rightarrow$ cell cycle coupling. As such, in Section 2 we study the entrainment of the cell cycle by the clock via the known molecular interaction whereby CLOCK:BMAL1 indirectly represses MPF (via the wee 1 gene [13]). In Section 3 we model and study the hypothesis of GF being not only a cell cycle input but also acting on the circadian clock system. This mechanism is explored in conjunction with the clock entrainment of the cell cycle via wee1 induction and results in a dynamic behavior that may help to explain the experimental observations of phase-locking synchronization dynamics ([5]). In this work, we observe 1:1, 3:2, 4:3 and 5:4 clock to cell cycle period ratios and identify GF and the coupling strength as decisive control parameters for the system's state of synchronization.

Moreover, we use the insight gained on the system's synchronization dynamics to explore strategies of period control. In particular, we focus on slowing down the cell cycle - an idea that may be of value for the control of cancer, which is characterized by a deregulated cell cycle. As such, we tune clock parameters and are able to obtain higher periods of clock and cell cycle in 1:1 synchronization as well as synchronization states with 2:3, 3:4 and 4:5 ratios of clock to cell cycle period, all cases consistent with a slower cell cycle. Our period control protocols create an understanding of multiple ways of slowing down the cell cycle, when making use of the coupled system synchronization state dynamics. Throughout the work, we observe the phase-locked oscillator, but focus mostly on observing variations of clock to cell cycle periods ratios, i.e. on the system's period-lock (synchronization) state.

\section{COUPLING VIA BMAL1-MEDIATED WEE1 ACTIVATION}

We start by establishing the basic dynamics of the unidirectional clock $\rightarrow$ cell cycle coupling. One notable mechanism is the induction of the wee1 gene by CLOCK:BMAL1 [13], as it involves the variable BMAL1 present in the clock model to be used [2] as well as the wee 1 interaction with MPF that is included in the cell cycle model [3]. Fig. 1 shows a scheme of the coupled system. Furthermore, Appendix A shows the equations of both models as well as parameters.

The action of WEE1 on MPF is included in the term $V_{w}$ ([3]), which is now multiplied by the variable BMAL1 to represent the effect of CLOCK:BMAL1 in promoting wee1 (and thus the repression of MPF). The equation for MPF now becomes: 


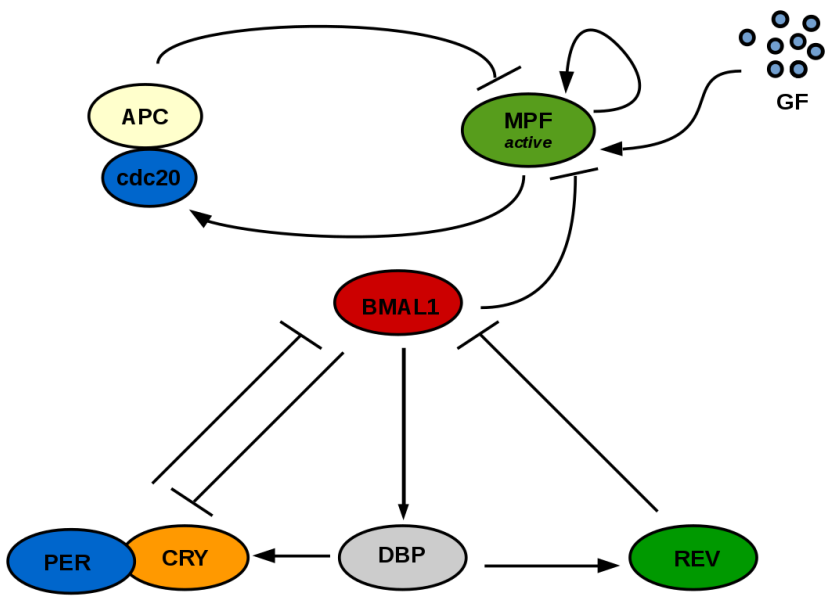

Figure 1: Schematic of the clock $\rightarrow$ cell cycle coupling mechanism. The CLOCK:BMAL1 protein complex (here the variable BMAL1) represses $M P F_{\text {active }}$ due to its action in promoting wee1 gene expression.

$$
\begin{aligned}
\frac{d[M P F]}{d t}= & G F+V_{c} \frac{\overline{M P F_{\max }}-[M P F]}{\overline{M P F_{\max }}-[M P F]+k_{c}} \frac{[M P F]^{2}}{[M P F]^{2}+k_{m}^{2}} \\
& -\mathbf{c}_{\mathbf{b}}[\mathrm{BMAL1}] V_{w} \frac{[M P F]}{[M P F]+k_{w}} \frac{k_{n}^{2}}{[M P F]^{n}+k_{n}^{2}} \\
& -\gamma_{1}[A P C: c d c 20][M P F]
\end{aligned}
$$

where $c_{b}$ is the coupling strength parameter. Fig. 2 shows an oscillatory solution of the main cell cycle variable MPF and the main clock variable BMAL1. For GF $=10$ and $c_{b}=10$ the clock entrains the cell cycle to its intrinsic period of $24 \mathrm{~h}$. Because both clock and cell cycle systems were previously normalized to a certain concentration value [2] [3] , the solution of the coupled system is dimensionless.

We will now observe how control parameters affect the synchronization state. Fig. 3 shows the effect of GF on the synchronization ratios for fixed $c_{b}=10$ : for higher GF the ratio of clock to cell cycle period $\left(r_{T}=\frac{T_{\text {clock }}}{T_{\text {cell cycle }}}\right)$ tends to increase.

Furthermore, Table 1 shows the system's period-lock response to variation of the control parameter $c_{b}$ for fixed GF, which predicts the appearance of 4:3 and 5:3 synchronization states; $\mathrm{GF}=40$ requires a much higher value of $c_{b}$ for $1: 1$ synchronization than $\mathrm{GF}=20$. For $\mathrm{GF}=20$, the $4: 3$ and $1: 1$ period-lock ratios are obtained, while $\mathrm{GF}=$ 40 results in 5:3, 3:2 and 1:1 synchronization.

Moreover, in Fig. 4 the periods of both oscillators are shown for $c_{b}=10$, for the same simulation as Fig. 3 . Note that only the cell cycle is responsive to GF. Thus, as expected for the clock $\rightarrow$ cell cycle unidirectional coupling, the period of the clock is kept at $24 \mathrm{~h}$, while the period of the cell cycle is sped up. This results in regions of a slower clock than cell cycle, which is in accordance with experimental observations [5].

A question of interest is the possibility of tuning the period of each oscillator by using the knowledge gained on the coupled

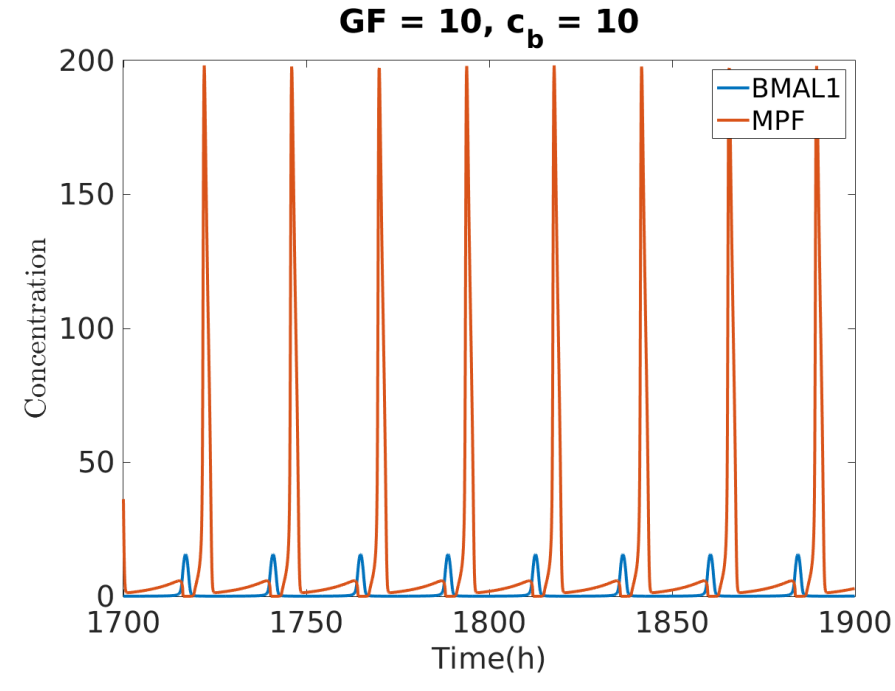

Figure 2: Oscillation of MPF and BMAL1. For GF $=10$ and $c_{b}$ $=10$ the system oscillates with a $24 \mathrm{~h}$ period.

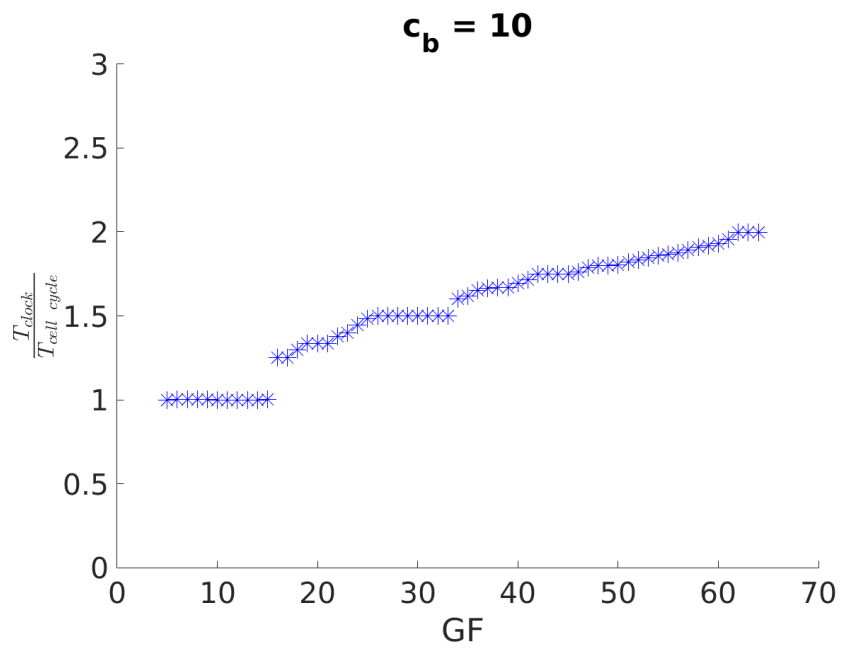

Figure 3: GF controls the synchronization ratio of the coupled system that increases with GF.

system. In particular, the ability of slowing down the cell cycle using clock inputs is relevant for cancer treatment. From Fig. 4, this may be achieved by either promoting a slower clock together with coincidental period ratios $\left(r_{T}=1\right)$ or hypothetically a faster clock with $r_{T}>1$ so as to observe if an adaptation of the cell cycle period occurs to a higher value (the opposite of what has been achieved in Fig. 4).

\subsection{Cell Cycle Period Control via the Clock}

In order to explore cell cycle period control via clock inputs, we focus on procedures that are experimentally feasible, such as single parameter changes. These have the potential to be reproduced 
Table 1: $c_{b}$ controls the system's synchronization ratio

\begin{tabular}{cc|cc}
\multicolumn{2}{c|}{$\mathrm{GF}=20$} & \multicolumn{2}{c}{$\mathrm{GF}=40$} \\
\hline$c_{b}$ & $r_{T}$ & $c_{b}$ & $r_{T}$ \\
\hline 4 & 1.33 & 40 & 1.67 \\
8 & 1.33 & 60 & 1.67 \\
12 & 1.33 & 80 & 1.67 \\
16 & 1.33 & 100 & 1.67 \\
20 & 1.33 & 120 & 1.67 \\
24 & 1.33 & 140 & 1.67 \\
28 & 1.33 & 180 & 1.67 \\
32 & 1.33 & 200 & 1.67 \\
36 & 1.33 & 220 & 1.67 \\
40 & 1.31 & 240 & 1.50 \\
44 & 1.00 & 260 & 1.50 \\
48 & 1.00 & 280 & 1.00 \\
52 & 1.00 & 300 & 1.00
\end{tabular}

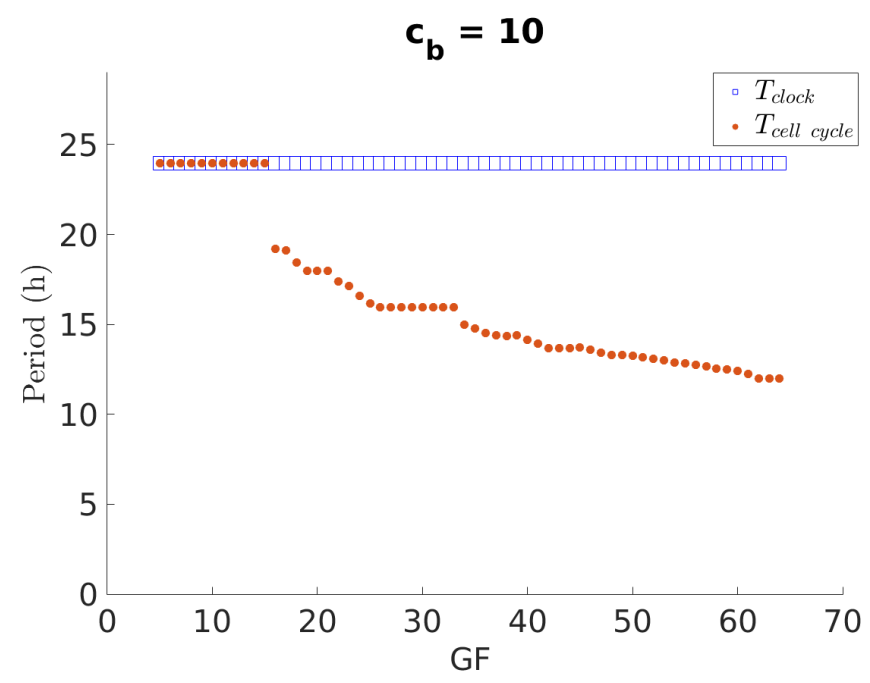

Figure 4: Circadian clock and cell cycle periods for $c_{b}=10$. The period of the clock (blue squares) doesn't change with GF in the unidirectional coupling, while the period of the cell cycle (red dots) adapts in a stepwise manner.

in experimental settings contingent upon the discovery of target molecular compounds that specifically affect them. From Almeida et al., [2] the parameters that affect clock period the most are those of R-box $\left(V_{R}\right.$ and $\left.k_{R r}\right)$ as well as the degradation rates of REV and DBP $\left(\gamma_{r e v}\right.$ and $\left.\gamma_{d b}\right)$. Thus, these are the best candidates for synchronization control analysis.

We start by introducing the parameter $\alpha$ in the R-box equation as: $R_{b o x}=V_{R} \frac{k_{R r}^{2}}{k_{R r}^{2}+(\alpha[R E V])^{2}}$. Here, $\alpha=1$ represents the original system oscillating with the intrinsic period, while the parameter $\alpha$ can either represent an R-box agonist (REV antagonist) for $\alpha<1$ or an R-box antagonist (REV agonist) for $\alpha>1$ by comparison with the control state. This application is identical to rescaling the parameter $k_{R r} \rightarrow \frac{k_{R r}}{\alpha}$. Fig. 5 shows how $\alpha$ affects the period of both oscillators and the ratio of clock to cell cycle period. When $\alpha \geq 1$ the system is naturally entrained with a $1: 1$ period-lock and increasing $\alpha$ increases the periods of both clock and cell cycle. When $\alpha<1$ higher values of R-box expression and shorter clock period are obtained resulting in a substantially increase in cell cycle period, in accordance with our hypothesis. Thus, both slowing down and speeding up the clock have the potential to slow down the cell cycle, the first method works by maintaining a strong coupling between the two oscillators and the second by accelerating the clock enough to drive the system away from the 1:1 region causing the adaptation of the cell cycle period.
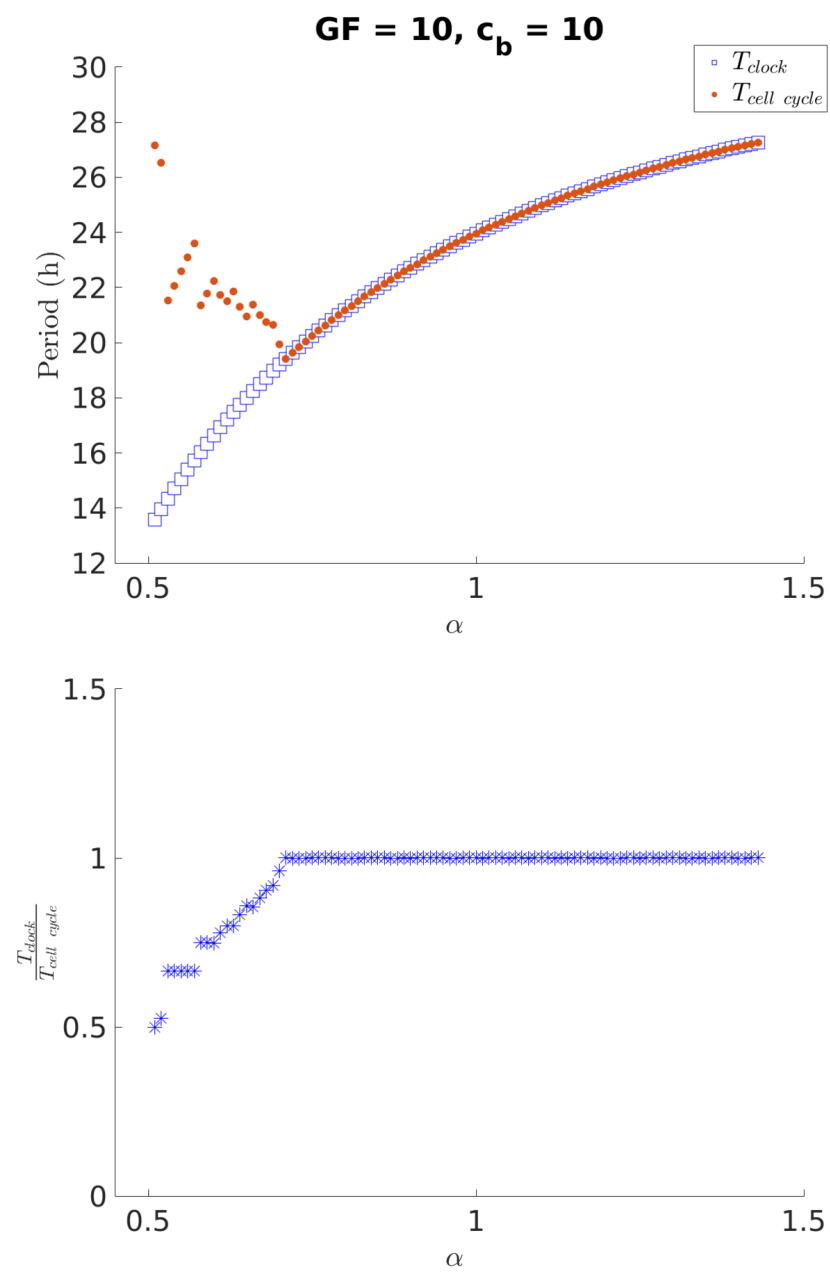

Figure 5: Evolution of the oscillators' period and synchronization ratio with $\alpha$. On top: values of $\alpha<1$ accelerate the clock (blue) and for a region of small $\alpha$ the cell cycle (red) "adapts" by slowing down, which makes the ratio between the two periods smaller than 1 (bottom); for $\alpha \geq 1$ the system synchronizes in a 1:1 manner and increasing the clock's period also increases that of the cell cycle. Overall, the 1:1, 1:2, 2:3, 3:4 and 4:5 ratios are obtained. 
Next, we observe period control via tuning of the REV and DBP rates of natural degradation, $\gamma_{r e v}$ and $\gamma_{b p}$. Some available compounds are known to interfere with $\gamma_{r e v}$ that could thus be used for tuning this parameter. These drugs act mostly via inhibition of GSk3 $\beta$, known to increase phosphorylation of REV-ERB, and can lead either to a decreased or increased clock period [19] depending on the GSK-inhibitor used [10] [21]. We thus introduce $\beta$ and $\delta$ as modulators of these parameters as: $\gamma_{r e v} \rightarrow \beta \gamma_{r e v}$ and $\gamma_{d b} \rightarrow \delta \gamma_{d b}$. Fig. 6, shows a solution of the system with a combination of increased $\gamma_{r e v}$ and $\gamma_{d b}$. In this case, $\beta=2$ and $\delta=1.5$ leads to a much slower cell cycle overall $(\mathrm{T}=31.5 \mathrm{~h})$ and a 1:3 period entrainment.

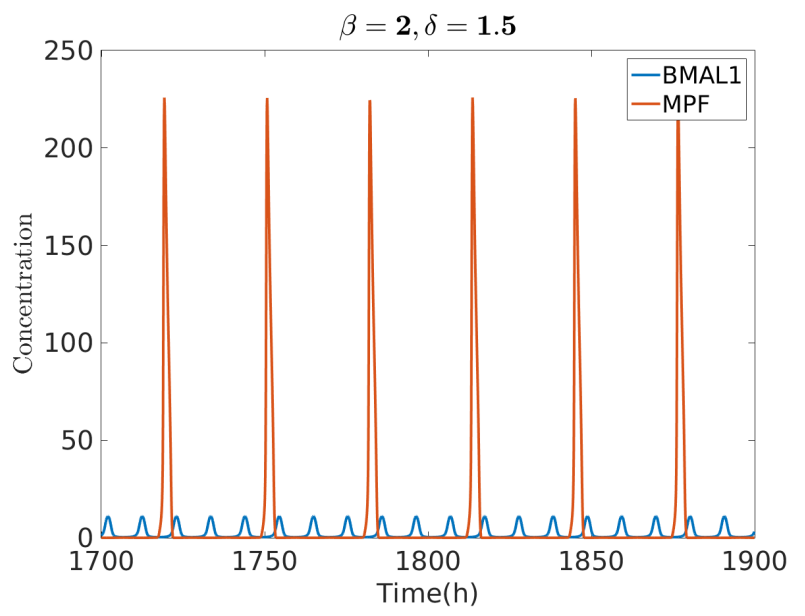

Figure 6: Oscillation of clock and cell cycle variables for $\beta=2, \delta=1.5, \mathbf{G F}=10$ and $c_{b}=10$. The system locks in a 1:3 ratio: $\mathrm{T}_{\text {clock }}=10.5 \mathrm{~h}$ and $\mathrm{T}_{\text {cell cycle }}=31.5 \mathrm{~h}$. The cell cycle is $\mathbf{3}$ times slower than the clock and overall slower than the intrinsic coupled oscillators' period (for $\beta=1$ and $\delta=1$ ) of 24 h (see Fig. 2).

As we have focused on controlling the cell cycle because of its relation to cancerous cell's division rate, it is important to note that healthy cells are likely to have same period clock and cell cycle internal oscillators and the state of $r_{T} \neq 1$ may be an indicator or precursor of cellular disease [6]. This idea highlights the relevance of the coupling strength parameter that promotes 1:1 synchronization of clock and cell cycle. Thus, the experimental exploration of chemical compounds for the tuning of this parameter would be of relevance in this context.

\section{COUPLING VIA GF-INDUCED INHIBITION OF R-BOX}

In this Section we propose a different additional mechanism for the coupling of the mammalian clock and cell cycle. One of our goals is to reproduce and understand the dynamical interactions behind the phase-lock experimental observations of Feillet et al., [5] showing that increasing amounts of GF speed up both the cell cycle and the clock in a 1:1 synchronization state. So far, the hypothesis has been that the cell cycle exerts influence on the clock and studies have been performed on this assumption $[1,18]$. Here, however we study GF as a common input to both oscillators. Thus, instead of assuming a coupling hypothesis where GF acts on the cell cycle that in turn acts on the clock, we take a look at a pathway connecting GF with the clock, such that GF is included in the model as a direct clock input.

Growth factors promote expression of cyclin D, a non-essential cell cycle cyclin that is active when in a complex with either CDK4 or CDK6, via $\beta$-catenin mediated pathways [20]. Despite cyclin D being approximately constant during the cell cycle, its action during the G1 phase precedes the activation of subsequent cyclin-CDK complexes. However, because cyclin D is non-essential (its deletion mutants still have a functioning cell cycle) and considering cells can't divide without GF, there must be other ways for GF to affect the essential cell cycle elements, namely the cyclin B-CDK1 complex (MPF). In the cell cycle model here used ([3]) MPF responds directly to GF - an approximation that allows focusing on this exclusively essential cell cycle species.

The cyclin D-CDK4 complex is known to negatively regulate PGC1- $\alpha$, by promoting its repressor GCN5 [11]. PGC1- $\alpha$ is an important clock component, with a role in promoting binding of ROR to R-box. As such, we study the potential pathway connecting GF to the clock: GF $\rightarrow$ cyclinD-CDK4 $\rightarrow$ GCN5 $-\mid$ PGC1- $\alpha$. The effect of GF as a repressor of R-box via the cyclin D/PGC1- $\alpha$ pathway can be introduced by making the change $V_{R} \rightarrow V_{R} \frac{k_{s}}{k_{s}+G F}$. R-box now becomes:

$$
R_{\text {box }}=V_{R}\left(\frac{k_{s}}{k_{s}+G F}\right) \frac{k_{R r}^{2}}{k_{R r}^{2}+[R E V]^{2}}
$$

The hypothesis introduced in Equation 2 raises the question of whether or not a certain amount of GF is needed for clock oscillation, as cells in experimental settings usually require some growth factor (often in the form of \% of FBS) to be alive and functional. Because the clock model has been built and calibrated based on the established assumption of a cell autonomous clock, the removal of GF (from Equation 2) doesn't affect clock oscillations. Nevertheless, if that were to be the case, this could be achieved by adjusting the parameter $V_{R}$ to a higher value, incompatible with oscillations at $\mathrm{GF}=0$, and then modulate $V_{R}$ by the presence of GF (see Equation 2). However, for simplicity, we will assume GF as a clock input that can control its period but it's still not required for oscillation. This modeling implies that GF $=0$ yields the $24 \mathrm{~h}$ intrinsic clock oscillation that has been the basis of our clock studies so far.

We verify that with this model, clock oscillations are maintained in the entire GF region of cell cycle oscillation $(4 \leq \mathrm{GF} \leq 80)$ and as GF increases the period of the circadian clock decreases. Thus, GF has a similar speeding up effect in both the clock and the cell cycle, which is in agreement with observations [5]. A schematic of the coupled system that includes the established interaction of BMAL1 repression of MPF via wee1 is shown in Fig. 7. The unidirectional clock $\rightarrow$ cell cycle coupling introduced in the previous section is maintained and GF is now a common input.

We start by analyzing the effect of GF on the oscillators' synchronization state for two values of $c_{b}$ with and without a Dexamethasone input. Fig. 8 (top) shows that for $c_{b}=10$ a region of 1:1 period-lock is followed by a region where the ratio of clock to cell cycle period increases up to a value of 1.3 . This synchronization 


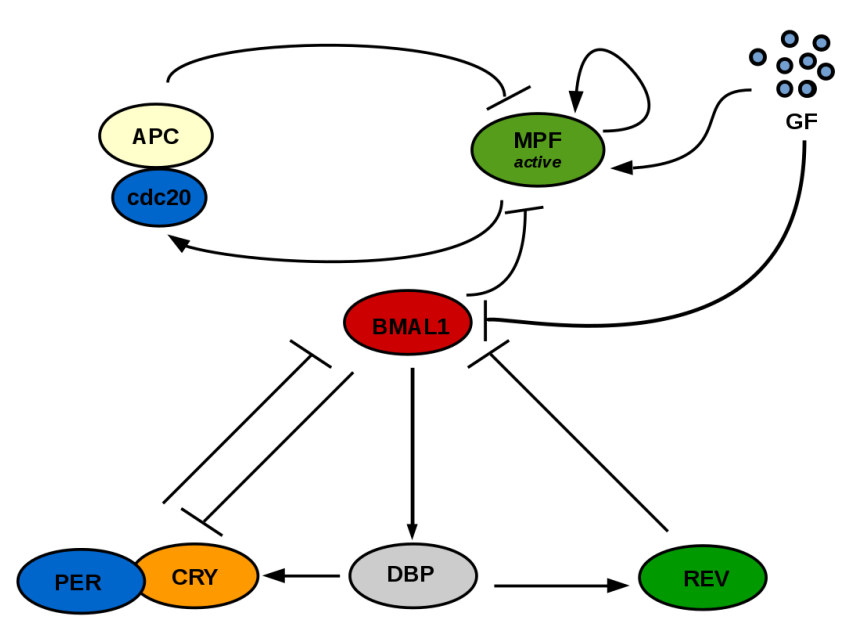

Figure 7: Schematic of the GF-responsive clock system coupled by the BMAL1 repression of active MPF (via the wee1 pathway). GF controls both the cell cycle and the clock. The hypothesis is that GF represses R-box (at the BMAL1 promoter) via the cyclin D-CDK4/PGC1 $\alpha$ pathway.

ratio adequately compares with the experimental observations of Feillet et al., [5]. By contrast, $c_{b}=50$ results in a wider region of 1:1 period-lock. Moreover, a Dex additive input on the PER equation (see Equation 6 of Appendix A) alters the system's behavior. In particular, for $c_{b}=10$ the $1: 1$ synchronization region becomes narrower with the point of synchronization state change being shifted to a lower value of GF (Fig. 8 (bottom)). This "shift to the left" effect is similar to that observed for Dex application in unidirectional cell cycle $\rightarrow$ clock and bidirectional coupling studied by Almeida, [1] and evidences a GF region where the presence of Dex drives the system away from the 1:1 synchronization state (an example would be GF $=20$, with $c_{b}=10$ ). Thus, assuming GF as a clock input in conjunction with the unidirectional clock $\rightarrow$ cell cycle coupling is also successful in reproducing experimental observations and may provide a complementary explanation for the coupled oscillators' behavior to that of a cell cycle direct action on the clock.

Moreover, we investigate the local maximum of $r_{T}$ in the region that follows the 1:1 synchronization (Fig. 8). On Table 2 we observe the maximum $r_{T}$ value for $4 \leq G F \leq 45$ for different values of $c_{b}$ with and without Dex. Without Dex, synchronization ratios vary between $5: 4$ and 4:3, specifically 1.3 for $c_{b}=10$, $4: 3$ for $c_{b}=20$ and $c_{b}=30$ and 5:4 for $c_{b}=40$. The presence of Dex lowers the maximum $r_{T}$ value of the system.

Additionally, Fig. 9 shows the period of the system as GF varies, for $c_{b}=10$ with no Dex. Differently from Fig. 4, the period of the clock is now responsive to GF. Nevertheless, observe that the GF region where the ratio between periods is kept close to a constant results from the adaptation of the cell cycle period to that of the clock, as expected for the unidirectional clock $\rightarrow$ cell cycle coupling. Thus, similarly to Section 2 , this raises the question of the possibility of cell cycle period control via clock tuning.
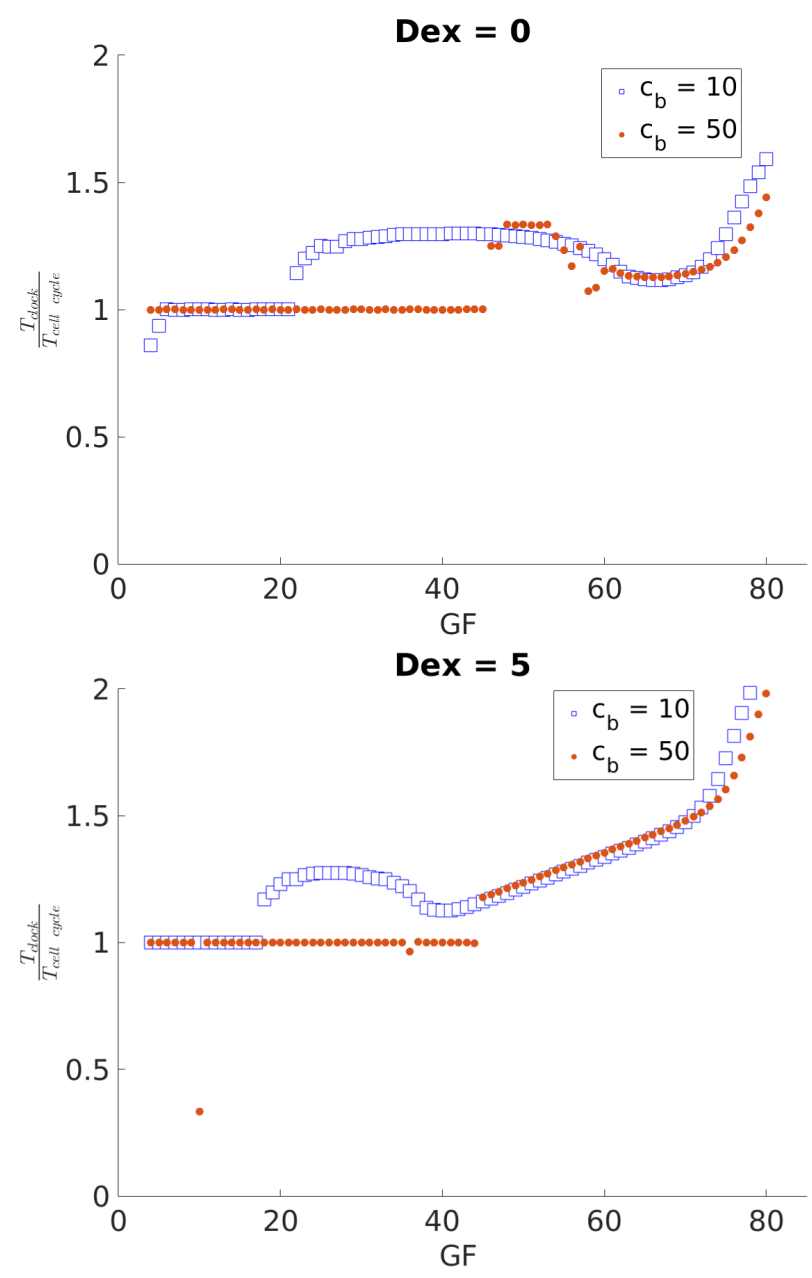

Figure 8: Variation of the synchronization ratio with GF for two values of $c_{b}$ with Dex $=0$ and Dex $=5$.

Table 2: Maximum synchronization ratio with $c_{b}$ for $4 \leq \mathrm{GF} \leq 45$, with Dex $=0$ and Dex $=5$.

\begin{tabular}{ccc}
$c_{b}$ & Dex $=0$ & Dex $=5$ \\
\hline 10 & 1.30 & 1.28 \\
20 & 1.33 & 1.29 \\
30 & 1.33 & 1.25 \\
40 & 1.25 & 1.18
\end{tabular}

We test this possibility by making $\gamma_{r e v} \rightarrow \beta \gamma_{r e v}$, as above, and varying $\beta$ for values around 1 , see Fig. 10 . In this case, we can vary $0.6 \leq \beta \leq 2.5$ and observe that slowing down the clock by decreasing $\beta$ effectively slows down the cell cycle while maintaining 1:1 synchronization. For $1<\beta<1.4$ we observe that speeding up the clock results in a sped up cell cycle, as the oscillators maintain the $1: 1$ synchronization, and for $\beta \geq 1.4$ the system breaks out of the 1:1 synchronization state and states of $r_{T}<1$ appear again, where the cell cycle is slower than the clock. Thus, tuning the 
parameter $\gamma_{r e v}$ is also successful in cell cycle period control in the unidirectional clock to cell cycle coupling with a GF-responsive circadian clock.

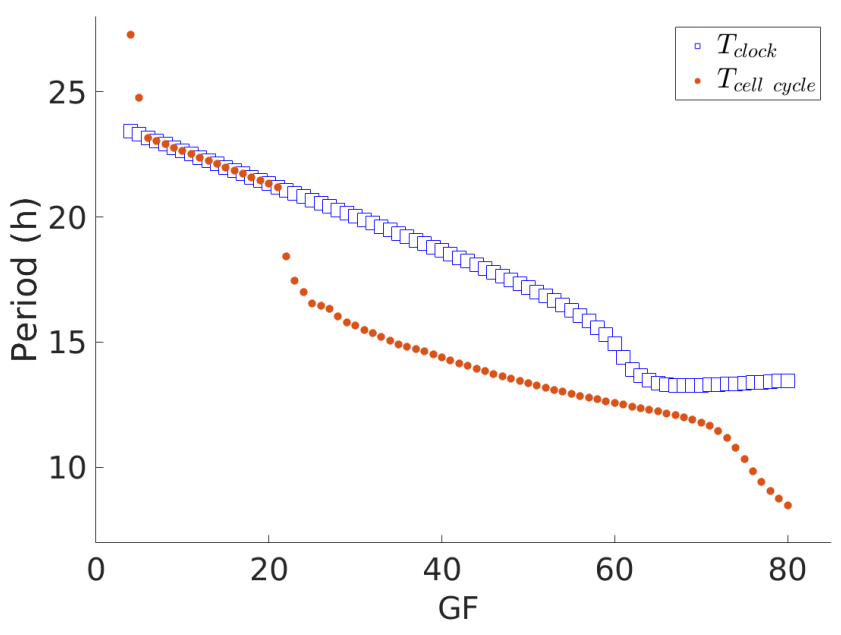

Figure 9: Periods of the GF-responsive clock and cell cycle as GF varies for $c_{b}=10$ and Dex $=0$.

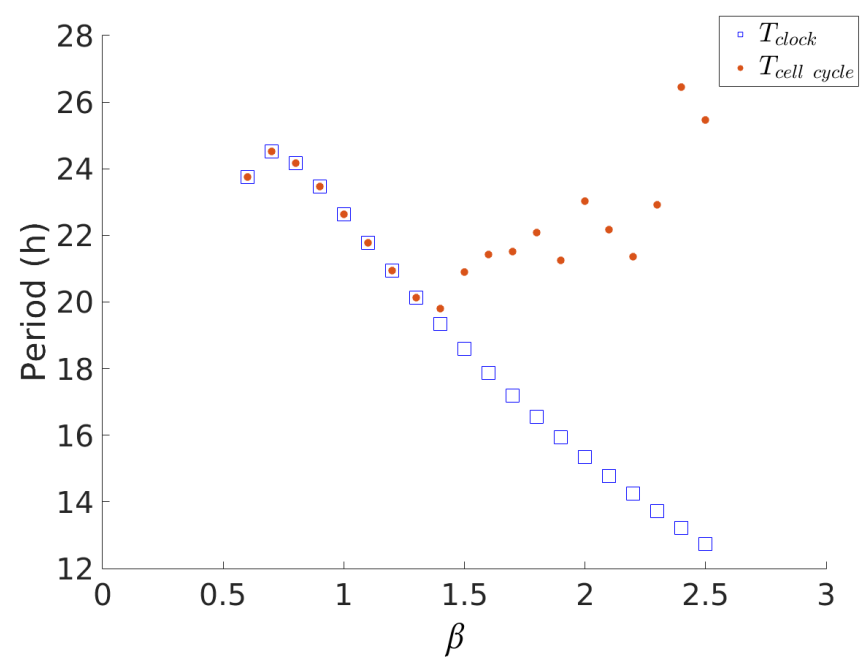

Figure 10: Variation of the GF-responsive clock and cell cycle periods with $\beta$. Slowing down the cell cycle is possible either by slowing down the clock, which maintains the 1:1 synchronization, or by speeding up the clock enough so as to cause the adaptation of the synchronization ratio to $r_{\mathrm{T}}<1$, resulting in a slowing down of the cell cycle by comparison with the control (with $\beta=1$ ).

In general, the hypothesis of a GF-responsive clock here proposed adds another layer of complexity to the coupling problem, as there isn't yet a way of knowing how relevant to circadian rhythms the effect of GF-transducing pathways is versus the effect of the cell cycle and whether this is influenced by the cellular (and extracellular) context (metabolism, signaling). In particular, the pathway proposed by us on Section 3 could be tested via knock-out experiments of the intermediary components cyclin D/CDK4 and GCN5. Moreover, experimental observations where GF is varied in a large interval with and without Dex (or other period tuning inputs) would allow comparison with our simulations and to understand which interactions are more relevant for the behavior of the system.

\section{CONCLUSIONS}

In this work we have studied unidirectional clock $\rightarrow$ cell cycle coupling centered on the observed CLOCK:BMAL1-induced wee1 activation, which was modeled as an MPF repression. Furthermore, we have modeled this interaction together with a GF action on the clock via a pathway involving the non-essential cell cycle complex cyclin D/CDK4. In both cases we have obtained ratios of clock to cell cycle periods compatible with experimental observations [5] and observed dynamical behavior under changes in coupling strength and GF. Furthermore, Dex application in the unidirectional clock $\rightarrow$ cell cycle coupling with a clock that is responsive to GF results in inducing $r_{T}>1$, also in accordance with observations [5].

From a biological a biological point of view, an exclusively unidirectional clock $\rightarrow$ cell cycle coupling mechanism implies that in order to obtain experimental synchronization ratios of accelerating clock and cell cycle with increasing GF, there needs to be a GF responsive clock. A bidirectional coupling on the other hand requires simultaneous modeling of both clock $\rightarrow$ cell cycle and cell cycle $\rightarrow$ clock molecular interactions, which raises questions from the modeling point of view as the latter hasn't been established. Almeida, [1] propose an action of MPF in phosphorylating an essential clock component, an hypothesis that also yields synchronization ratios comparable to experimental observations.

Moreover, we have explored cell cycle period control, with relevance for cancer growth control, by means of single-parameter changes in the clock system. We have found that it is possible to slow down the cell cycle by either slowing down the clock when promoting a state of 1:1 synchronization, or by speeding up the clock sufficiently so as to generate a state where the cell cycle adapts by slowing down maintaining a rational synchronization ratio. The methods here developed may form a basis for the creation of experimental protocols to test the synchronization dynamics of these oscillators.

\section{A MAMMALIAN CLOCK AND CELL CYCLE MODELS}

In this work we study the coupling of the mammalian cell cycle model developed by Almeida et al., [3] with the mammalian circadian clock model developed by Almeida et al., [2] and Almeida, [1]. The model equations are summarized here:

$$
\begin{gathered}
\frac{d[B M A L 1]}{d t}=R_{b o x}-\gamma_{b p}[B M A L 1][P E R: C R Y] \\
\frac{d[D B P]}{d t}=V_{B}[B M A L 1]-\gamma_{d b}[D B P] \\
\frac{d[R E V]}{d t}=V_{D 2}[D B P]-\gamma_{r e v}[R E V]
\end{gathered}
$$




$$
\begin{gathered}
\frac{d[P E R: C R Y]}{d t}=\operatorname{Dex}+V_{D 2}[D B P]-\gamma_{b p}[B M A L 1][P E R: C R Y] \\
\frac{d[M P F]}{d t}= \\
\quad G F+V_{c} \frac{\overline{M P F_{\max }}-[M P F]}{\overline{M P F_{\max }}-[M P F]+k_{c}} \frac{[M P F]^{2}}{[M P F]^{2}+k_{m}^{2}} \\
-V_{w} \frac{[M P F]}{[M P F]+k_{w}} \frac{k_{n}^{2}}{[M P F]^{n}+k_{n}^{2}} \\
-\gamma_{1}[A P C: c d c 20][M P F] \\
\frac{d[A P C: c d c 20]}{d t}=V_{m}[M P F]-V_{k}[A P C: c d c 20]
\end{gathered}
$$

where

$$
R_{\text {box }}=V_{R} \frac{k_{R r}^{2}}{k_{R r}^{2}+[R E V]^{2}}
$$

and the parameter GF represents an MPF synthesis term due to the presence of Growth Factor. BMAL1 represents the CLOCK:BMAL1 complex, REV all REV-ERBs and DBP all D-box activators. Parameters for all simulations are in Table 3 and we use the initial condition: $\mathrm{BMAL}=1.2 ; \mathrm{DBP}=1.6 ; \mathrm{REV}=1.5 ; \mathrm{PER}: \mathrm{CRY}=1.2 ; \mathrm{MPF}=2.0$; APC: $\operatorname{cdc} 20=1.0$. The Dex additive input to PER:CRY represents the hormone Dexamethasone and is used only once in Section 3, being kept at zero for the remaining studies.

Table 3: Parameters of the clock and cell cycle models

\begin{tabular}{cc}
$\mathrm{p}$ & Numerical Value \\
\hline$V_{R}$ & $34.4 \mathrm{~h}^{-1}$ \\
$k_{R r}$ & 80.1 \\
$V_{B}$ & $0.11 \mathrm{~h}^{-1}$ \\
$V_{D 2}$ & $14.7 \mathrm{~h}^{-1}$ \\
$\gamma_{\text {rev }}$ & $0.187 \mathrm{~h}^{-1}$ \\
$\gamma_{d b}$ & $0.121 \mathrm{~h}^{-1}$ \\
$\gamma_{b p}$ & $2.0 \mathrm{~h}$ \\
\hline$\gamma_{1}$ & $0.162 \mathrm{~h}^{-1}$ \\
$V_{c}$ & $2260 \mathrm{~h}^{-1}$ \\
$k_{c}$ & 130 \\
$V_{w}$ & $7480 \mathrm{~h}^{-1}$ \\
$k_{w}$ & 138 \\
$k_{m}$ & 99 \\
$k_{n}$ & 0.116 \\
$V_{m}$ & $1.68 \mathrm{~h}^{-1}$ \\
$V_{k}$ & $1.07 \mathrm{~h}^{-1}$ \\
\hline$M P F_{\max }$ & 284 \\
\hline
\end{tabular}

\section{ACKNOWLEDGMENTS}

The authors are part of the Labex SIGNALIFE Network for Innovation on signal Transduction Pathways in Life Sciences (Grant ANR-11-LABX-0028-01) and ICycle (ANR-16-CE33-0016-01).

\section{REFERENCES}

[1] Sofia Almeida. 2018. Synchronization of biological oscillators: modeling, analysis and coupling of the mammalian cell cycle and circadian clock. Ph.D. Dissertation. Université Côte d'Azur.

[2] Sofia Almeida, Madalena Chaves, and Franck Delaunay. 2019. Transcriptionbased circadian mechanism controls the duration of molecular clock states in response to signaling inputs. Journal of Theoretical Biology 484 (2019), 110015.

[3] Sofia Almeida, Madalena Chaves, Franck Delaunay, and Celine Feillet. 2017. A comprehensive reduced model of the mammalian cell cycle. IFAC-PapersOnLine 50, 1 (2017), 12617-12622.

[4] Jonathan Bieler, Rosamaria Cannavo, Kyle Gustafson, Cedric Gobet, David Gatfield, and Felix Naef. 2014. Robust synchronization of coupled circadian and cell cycle oscillators in single mammalian cells. Mol Syst Biol. 15, 10 (2014), 739.

[5] Céline Feillet, Peter Krusche, Filippo Tamanini, Roel C Janssens, Mike J Downey, Patrick Martin, Michèle Teboul, Shoko Saito, Francis A Lévi, Till Bretschneider, Gijsbertus T J van der Horst, Franck Delaunay, and David A Rand. 2014. Phase locking and multiple oscillating attractors for the coupled mammalian clock and cell cycle. Proceedings of the National Academy of Sciences of the United States of America 111, 27 (Jul 2014), 9828-33. https://doi.org/10.1073/pnas.1320474111

[6] Loning Fu and Cheng Chi Lee. 2003. The circadian clock: pacemaker and tumour suppressor. Nature Reviews Cancer 3, 5 (2003), 350.

[7] Loning Fu, Helene Pelicano, Jinsong Liu, Peng Huang, and Cheng Chi Lee. 2002. The circadian gene Period2 plays an important role in tumor suppression and DNA damage response in vivo. cell 111, 1 (2002), 41-50.

[8] Claude Gérard and Albert Goldbeter. 2012. Entrainment of the mammalian cell cycle by the circadian clock: modeling two coupled cellular rhythms. PLoS computational biology 8, 5 (May 2012), e1002516. https://doi.org/10.1371/journal. pcbi.1002516

[9] Aline Gréchez-Cassiau, Béatrice Rayet, Fabienne Guillaumond, Michèle Teboul, and Franck Delaunay. 2008. The circadian clock component BMAL1 is a critical regulator of p21WAF1/CIP1 expression and hepatocyte proliferation. Fournal of Biological Chemistry 283, 8 (2008), 4535-4542.

[10] Tsuyoshi Hirota, Warren G Lewis, Andrew C Liu, Jae Wook Lee, Peter G Schultz, and Steve A Kay. 2008. A chemical biology approach reveals period shortening of the mammalian circadian clock by specific inhibition of GSK- $3 \beta$. Proceedings of the National Academy of Sciences 105, 52 (2008), 20746-20751.

[11] Yoonjin Lee, John E Dominy, Yoon Jong Choi, Michael Jurczak, Nicola Tolliday, Joao Paulo Camporez, Helen Chim, Ji-Hong Lim, Hai-Bin Ruan, Xiaoyong Yang, et al. 2014. Cyclin D1-Cdk4 controls glucose metabolism independently of cell cycle progression. Nature 510, 7506 (2014), 547.

[12] Rachel Leproult, Ulf Holmbäck, and Eve Van Cauter. 2014. Circadian misalignment augments markers of insulin resistance and inflammation, independently of sleep loss. Diabetes 63, 6 (2014), 1860-1869.

[13] Takuya Matsuo, Shun Yamaguchi, Shigeru Mitsui, Aki Emi, Fukuko Shimoda, and Hitoshi Okamura. 2003. Control mechanism of the circadian clock for timing of cell division in vivo. Science 302, 5643 (2003), 255-259.

[14] Emi Nagoshi, Camille Saini, Christoph Bauer, Thierry Laroche, Felix Naef, and Ueli Schibler. 2004. Circadian gene expression in individual fibroblasts: cellautonomous and self-sustained oscillators pass time to daughter cells. Cell 119, 5 (2004), 693-705.

[15] Narin Ozturk, Dilek Ozturk, Ibrahim Halil Kavakli, and Alper Okyar. 2017. Molecular aspects of circadian pharmacology and relevance for cancer chronotherapy. International journal of molecular sciences 18, 10 (2017), 2168.

[16] Ignacio Pérez-Roger, David LC Solomon, Andreas Sewing, and Hartmut Land. 1997. Myc activation of cyclin E/Cdk2 kinase involves induction of cyclin E gene transcription and inhibition of p27 Kip1 binding to newly formed complexes. Oncogene 14, 20 (1997), 2373.

[17] Anton Shostak. 2017. Circadian clock, cell division, and cancer: from molecules to organism. International journal of molecular sciences 18, 4 (2017), 873.

[18] Pauline Traynard, Céline Feillet, Sylvain Soliman, Franck Delaunay, and François Fages. 2016. Model-based investigation of the circadian clock and cell cycle coupling in mouse embryonic fibroblasts: prediction of RevErb- $\alpha$ up-regulation during mitosis. Biosystems 149 (2016), 59-69.

[19] Thomas Wallach and Achim Kramer. 2015. Chemical chronobiology: toward drugs manipulating time. FEBS letters 589, 14 (2015), 1530-1538.

[20] Ping Ye, Qichen Hu, Hedi Liu, Yun Yan, and A Joseph D'ercole. 2010. $\beta$-catenin mediates insulin-like growth factor-I actions to promote cyclin D1 mRNA expression, cell proliferation and survival in oligodendroglial cultures. Glia 58, 9 (2010), 1031-1041.

[21] Lei Yin, Jing Wang, Peter S Klein, and Mitchell A Lazar. 2006. Nuclear receptor Rev-erb $\alpha$ is a critical lithium-sensitive component of the circadian clock. Science 311, 5763 (2006), 1002-1005

[22] Judit Zámborszky, Christian I Hong, and Attila Csikász Nagy. 2007. Computational analysis of mammalian cell division gated by a circadian clock: quantized cell cycles and cell size control. fournal of biological rhythms 22, 6 (Dec 2007), 542-53. 